\title{
Influence of span-wise coherence on the acoustic radiation in a cylinder wake
}

\author{
Lars Siegel $^{1}$, Guosheng He ${ }^{2,3}$, Karen Mulleners ${ }^{2}$, Arne Henning ${ }^{1 *}$ \\ ${ }^{1}$ German Aerospace Center (DLR), Inst. for Aerodyn. \& Flow Tech., Göttingen, Germany \\ ${ }^{2}$ Institute of Mechanical Engineering, École polytechnique fédérale de Lausanne (EPFL), Switzerland \\ ${ }^{3}$ School of Astronautics, Beijing Institute of Technology, China \\ *arne.henning@dlr.de
}

\begin{abstract}
The aim of this study is to detect and visualise the influence of span-wise coherence on propagating sound waves emanating from a flow around circular cylinders with span-wise variations of the local radius. Synchronous particle image velocimetry (PIV) and microphone measurements are performed in a circular wind tunnel with a nozzle size of $0.4 \mathrm{~m} \times 0.4 \mathrm{~m}$ at a maximum flow speed of $U_{\infty}=43 \mathrm{~m} \mathrm{~s}^{-1}$. The test section is surrounded by a full anechoic chamber of approximately $9 \mathrm{~m} \times 9 \mathrm{~m} \times 5 \mathrm{~m}$.

The two components (2C) of the velocity field data are acquired in selected vertical two-dimensional planes (2D) using 2C2D-PIV around the cylinder mid-span. All three velocity components (3C) are acquired using stereoscopic or 3C2D-PIV in a horizontal span- and flow-wise oriented plane in the near wake of the cylinder. The pressure measurements are conducted with 4 microphones (Type:1/4 40BF; G.R.A.S.) in the far field outside the flow to avoid unwanted influences on the flow field and vice versa. The microphones are installed above the cylinder and are distributed in a horizontal line at the mid-span of the cylinders in flow direction to take into account the directivity of the sound emission. The vertical positions were approximately $1.8 \mathrm{~m}(120 \mathrm{~d})$ above the cylinder and the distance between the microphones was about $0.3 \mathrm{~m}$ $(20 d)$. A multi-analyzer (Type: DEWE3; Dewetron) simultaneously records the microphone-signals, the camera trigger, the q-switch of the laser and three components of the forces acting on the cylinders with a sampling frequency of $f_{s}=100 \mathrm{kHz}$ and a dynamic range of 24 bit. Cylinders with different span-wise variations are investigated as illustrated in fig. 11. The geometric parameters of the cylinders are selected such
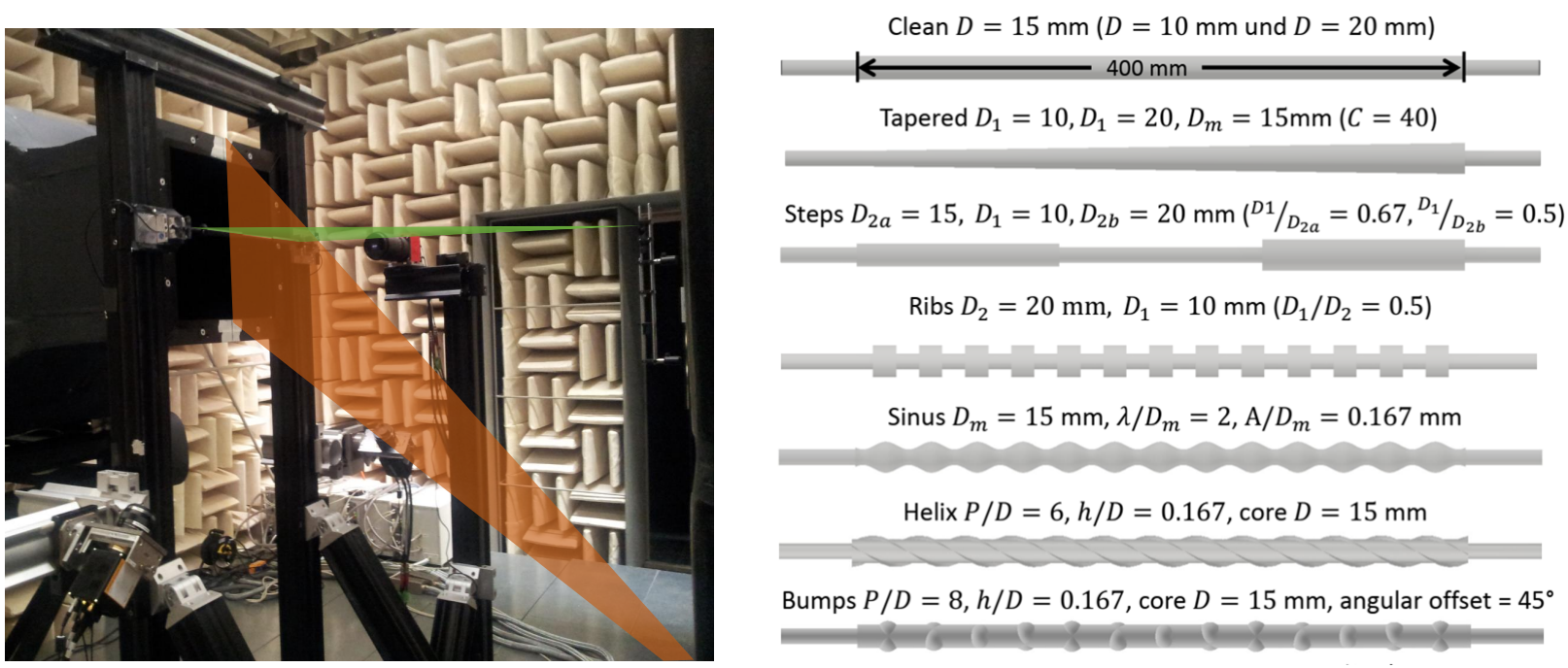

Bumps $P / D=8, h / D=0.167$, core $D=15 \mathrm{~mm}$, angular offset $=45^{\circ}$
\end{abstract}

Figure 1: Experimental setup with indicated light sheets for the 2C2D-PIV (orange) and 3C2D-PIV (green) (left); and the different cylinders tested (right). 

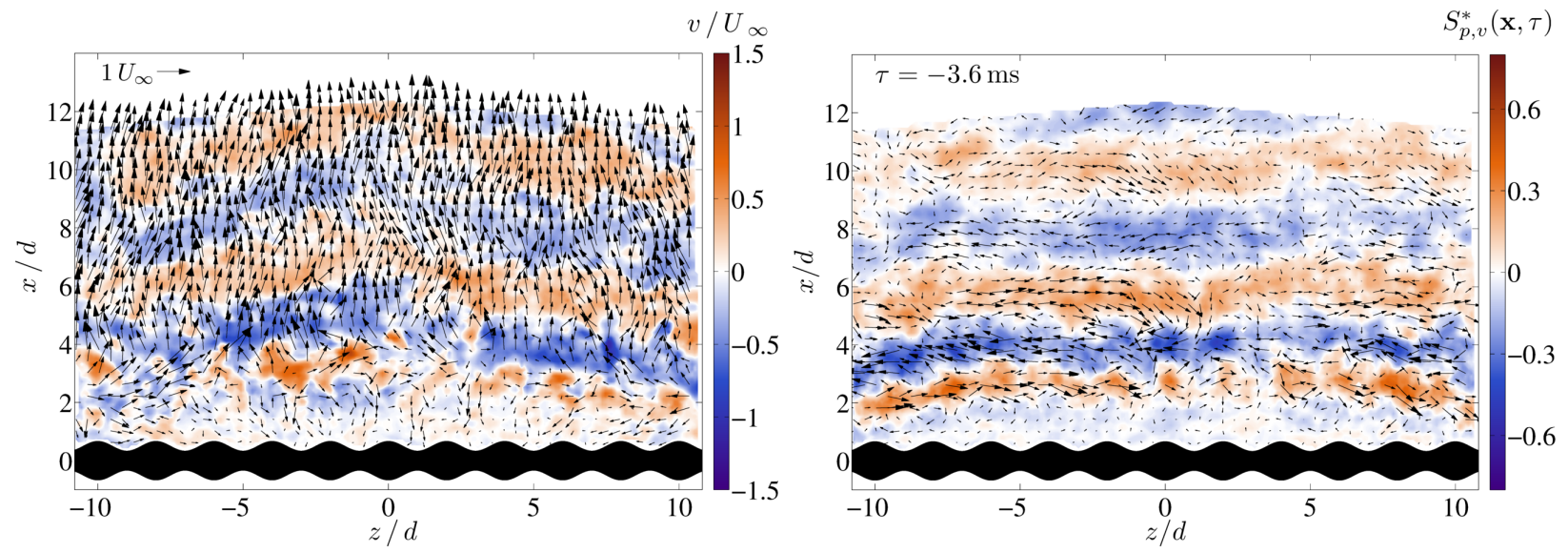

Figure 2: Instantaneous vector distributions of the velocity field (left) and the cross-correlation function (right) of the sinusoidal cylinder configuration in the horizontal plane. Color-coded is the out-of-plane component.

that the core or the average diameter $d$ is $15 \mathrm{~mm}$ and the span-wise wavelength for the repeated variations are constant.

Figure 2(left) shows an example of the instantaneous distribution of the velocity field of the sinusoidal cylinder configuration in the horizontal plane. The axis are scaled with the cylinder diameter $d$ and $U_{\infty}$ is the free-stream velocity. The out-of-plane component $v$ pointing into the direction of the microphones is color-coded. The span-wise coherent detachment of the vortex patterns are superimposed by turbulent motions produced by the interaction between smaller scale locally detaching vortices along the sinusoidal protrusions of the cylinder. By correlating the velocity $\phi^{\prime}$ and pressure fluctuations $p^{\prime}$ in the far field, we can identify the influence of the span-wise cylinder surface variations on the acoustic emission (Siegel et al. 2018; Henning et al. 2008). Figure 2(right) depicts the instantaneous distribution of the cross-correlation function which is defined as follows:

$$
S_{p, \phi}(\mathbf{x}, \tau)=\frac{1}{N} \sum_{i=1}^{N} p^{\prime}\left(t_{i}+\tau\right) \cdot \phi^{\prime}\left(\mathbf{x}, t_{i}\right),
$$

with $\tau$ the time shift between the velocity and pressure measurement. Again, the out-of-plane component is color-coded. The span-wise coherence becomes more apparent, while the wavelike characteristic of the coherent structures is a consequence of the sinusoidal variation along the span of the cylinder. The aim is to characterize the wake topologies behind the different cylinder geometries in detail and to determine the influence of the surface variations on the vortex shedding frequencies. The final analysis considers the acoustic signatures of the different topologies and the corresponding span-wise coherence. The analysis of the temporal and spatial evolution of these structures provides an insight into the sound generation mechanism.

\section{Acknowledgements}

This work was supported by the Swiss national science foundation Lead Agency programme under grant number 200021E-169841 and the Deutsche Forschungsgemeinschaft (DFG - German Research Foundation) under grant number HE 7369/2-1.

\section{References}

Henning A, Kaepernick K, Ehrenfried K, Koop L, and Dillmann A (2008) Investigation of aeroacoustic noise generation by simultaneous particle image velocimetry and microphone measurements. Experiments in Fluids 45:1073-1085

Siegel L, Ehrenfried K, Wagner C, Mulleners K, and Henning A (2018) Cross-correlation analysis of synchronized PIV and microphone measurements of an oscillating airfoil. Journal of Visualization 21 